\title{
Uso do Scratch no Brasil com objetivos educacionais: uma revisão sistemática
}

Adelmo Antonio da Silva Eloy, Universidade de São Paulo - adelmo.eloy@ usp.br

Roseli de Deus Lopes, Universidade de São Paulo - roseli.lopes@usp.br Isabela Martins Angelo, Universidade de São Paulo - isabela.angelo@usp.br

Resumo: O Scratch é uma linguagem de programação visual utilizada no mundo inteiro para introdução à programação, com mais de 18 milhões de usuários cadastrados. Nesse contexto, este artigo apresenta uma revisão sistemática da literatura em torno do uso do Scratch no Brasil com fins educacionais, identificando e analisando os artigos sobre o tema publicados em língua portuguesa em revistas e eventos, entre 2012 e 2016 . Por meio da revisão, foi possível caracterizar o público e contexto de uso do Scratch, que em sua maioria são estudantes do ensino fundamental - apesar de parcela considerável ser do ensino superior - em ambientes escolares, com o fim de promover a introdução à programação ou ao pensamento computacional. Não se observou uniformidade quanto à descrição de estratégias de avaliação e de impactos aferidos, mas em geral apontam como principais objetivos melhor desempenho acadêmico e maior engajamento escolar.

Palavras-chave: Scratch; Educação; Programação.

\section{Scratch in Brazil for educational purposes: a systematic review}

Abstract: Scratch is a visual programming language used worldwide for introducing programming, with more than 18 million users. In this context, this paper presents a systematic review about the use of Scratch in Brazil for educational purposes, identifying and analyzing articles on the topic in national journals and events, between 2012 and 2016. From this review, it was possible to characterize the public and context of use of Scratch, mostly students from elementary school - despite a considerable number of experiences in higher education - in school environments, in order to promote the introduction to Programming or Computational Thinking. The main results are not uniform in terms of strategies of evaluation and impacts, but usually involve academic performance and school engagement.

Keywords: Scratch, Education, Programming.

\section{Introdução}

O Scratch é uma linguagem de programação visual desenvolvida por equipe do MIT e lançada em 2007, contendo uma comunidade de usuários espalhada por todo o mundo. De acordo com dados de sua plataforma online, hoje estão registrados mais de 18 milhões de usuários (cerca de 276 mil do Brasil), e mais de 22 milhões de projetos compartilhados (Scratch, 2017). Ele foi desenvolvido com foco no público de 8 a 16 anos, mas hoje é utilizado por uma faixa etária muito mais ampla. Entre seus diferenciais, estão a facilidade de uso e diversidade de construções possíveis estimulando a criatividade, em concordância com a ideia de "piso baixo e teto alto" (poucas restrições para início de uso, mas possibilidades ilimitadas de aprofundamento) proposta por Papert (1980) e pelas "paredes amplas" (pluralidade de caminhos e temas) de Resnick et. al (2009).

Disponível por meio de plataforma web, pode ser acessado online ou offline, o Scratch torna a prática da programação acessível a diferentes públicos, permitindo que jogos e histórias interativas digitais sejam criados e compartilhados por meio da conexão de blocos intuitivos e próximos da língua falada. Mais do que uma ferramenta com foco em preparar as próximas gerações para o mercado de trabalho, seus criadores o enxergam 
como um recurso que contribui para a formação de pessoas criativas e com capacidade de sistematizar e expressar ideias por meio da programação (Resnick et al., 2009).

Esses e outros atributos do Scratch foram determinantes em sua popularização, o que resulta hoje em grande diversidade de usos relatados: integrado com áreas do conhecimento tradicionais e já consolidadas no ambiente escolar, como a Matemática (Foerster, 2016); associado a novas tendências, como Big Data e Internet das Coisas (Dasgupta; Resnick, 2014); como ferramenta de introdução à computação no ensino superior (Malan; Leitner, 2007); em contextos não esperados inicialmente, como a integração ao ambiente virtual Second Life com o objetivo de facilitar a criação nesse universo (Pellas; Peroutseas, 2016). Além disso, como apontam Bombasar et al. (2015), o Scratch pode ser considerado atualmente como a principal ou uma das principais ferramentas de ensino-aprendizagem de pensamento computacional. Esse cenário motiva, então, a análise de trabalhos de revisão que busquem entender mais a fundo como o Scratch tem sido utilizado com fins educacionais.

Contudo, a partir da análise de trabalhos de revisão sistemática recentes (MorenoLeón; Robles, 2016), nota-se um ponto interessante: a ausência de relatos de experiências realizadas no Brasil. Por outro lado, revisões da literatura nacional sobre as temáticas de pensamento computacional e programação, a linguagem Scratch aparece com destaque (Ferri; Santos Rosa, 2016) e encontram-se relatos de várias experiências de uso em diferentes contextos e públicos de faixa etária e nível escolar variados, reforçando a sua abrangência de uso no país. Uma possível justificativa para a ausência citada, então, vem a ser a barreira do idioma, dado que veículos nacionais, em sua maioria, divulgam os trabalhos em língua portuguesa e isso se torna um empecilho para que a comunidade acadêmica internacional conheça os trabalhos aqui produzidos.

Dessa forma, para entender melhor o cenário nacional, o presente trabalho apresenta uma revisão sistemática da literatura (RSL) em relação ao uso Scratch no Brasil com objetivos educacionais. A seção 2 apresenta trabalhos relacionados ao tema proposto. A seção 3 descreve o método utilizado na revisão. A seção 4 apresenta os resultados obtidos e uma discussão em torno dos mesmos. A seção 5 apresenta as considerações finais e sugestões de trabalhos futuros.

\section{Trabalhos relacionados}

Conforme descrito na Seção 1, a literatura sobre estudos e experiências utilizando o Scratch é vasta tanto em termos de quantidade como de temática. Além disso, é possível encontrar na literatura nacional e internacional revisões sistemáticas que reforçam sua relevância em diferentes contextos. A revisão proposta por Vihavainen, Airaksinen e Watson (2014) indica o Scratch como uma das mais comuns ferramentas na introdução à programação visual, estando em conjunto com Alice em 5 de 9 estudos selecionados; a revisão realizada por Bombasar et al. (2015) indica o Scratch como a principal ferramenta para ensino-aprendizagem de pensamento computacional, estando presente em 31 de 106 estudos selecionados. Moreno-León e Robles (2016), por outro lado, investigam como a introdução à programação de computadores com Scratch pode impactar outras áreas do conhecimento, identificando 15 trabalhos para análise, mas sua revisão não aborda trabalhos em língua portuguesa. Souza e Castro (2016), que se propõem a fazer uma revisão sistemática sobre o Scratch, têm em sua maioria publicações internacionais, não permitindo a análise do contexto nacional.

\section{Materiais e métodos}

A RSL aqui apresentada utilizou como referência a proposta metodológica de Kietchenham (2007), consistindo em três etapas: planejamento, condução e relato da 
revisão. O planejamento é composto pela definição das questões de pesquisa, construção da estratégia de busca e definição de critérios de exclusão, inclusão e avaliação, que serão apresentados conjuntamente com os resultados obtidos a partir da conduta da revisão. $\mathrm{O}$ período de busca definido foi de 5 anos, abrangendo de 2012 a 2016, pois, apesar de disponível desde 2007, a partir de 2012 foi criado o ambiente de criação e compartilhamento de projetos online, o que ampliou significativamente o seu uso.

\subsection{Questões de pesquisa}

Inicialmente foram definidas as questões de pesquisa, derivadas da questão principal "Como o Scratch tem sido explorado em experiências educacionais no Brasil?", as quais foram:

QP1: Quais são os objetivos de uso do Scratch nas pesquisas relatadas?

QP2: Quais são os contextos e público-alvo relatados?

QP3: Quais são as estratégias de avaliação e os resultados relatados?

QP4: Há instituições que se destacam nesse cenário?

\subsection{Estratégia de busca}

A busca de trabalhos para responder a essas perguntas abrangeu as cinco etapas descritas a seguir:

\subsubsection{Identificação de periódicos nacionais mais relevantes relacionados ao tema}

Por apresentar resultados de diferentes canais de divulgação científica nacionais, o Google Acadêmico foi utilizado para fazer a primeira busca de resultados, utilizando as palavras "Scratch" e "programação" (termo em português comumente associado ao Scratch nos contextos de interesse desta pesquisa). Os resultados utilizados foram analisados com o auxílio da ferramenta Zotero, que permite agrupar e organizar os resultados de busca obtidos. Utilizando o recurso da ferramenta que identifica o veículo de cada publicação, foi possível identificar os mais relevantes (considerados aqueles com no mínimo de 5 artigos), que são: Workshop de Informática na Escola (WIE), 49 artigos; Congresso Brasileiro de Informática na Educação (CBIE), 34 artigos; Simpósio Brasileiro de Informática na Educação (SBIE), 29 artigos; Revista Novas Tecnologias na Educação (RENOTE), 15 artigos; Revista Brasileira de Informática na Educação (RBIE), 8 artigos; e Jornada de Atualização em Informática na Educação (JAIE), 6 artigos.

\subsubsection{Busca de artigos nos periódicos e anais dos eventos selecionados}

Os periódicos e anais de eventos identificados possuem mecanismos de busca em seus respectivos portais; contudo, notou-se um número muito menor de resultados quando comparado ao resultado via Google Acadêmico e Zotero. Acredita-se que isso se deve ao fato dos mecanismos destes portais buscarem as palavras-chave apenas no título e resumo dos artigos. Por isso, optou-se por fazer uma busca manual nos periódicos e anais de eventos selecionados, utilizando a ferramenta de busca por palavras do Adobe Reader em arquivos no formato PDF, utilizando a palavra-chave "Scratch". A partir dessa busca foram encontrados 104 artigos, distribuídos conforme apresentado na tabela 1.

Tabela 1: Número de artigos com a palavra Scratch, por veículo, de 2012 a 2016

\begin{tabular}{|c|c|c|c|c|c|c|}
\hline Veículo & WIE & CBIE & SBIE & RENOTE & RBIE & JAIE \\
\hline Número de artigos & 33 & 22 & 20 & 18 & 8 & 3 \\
\hline No. após critérios & 21 & 13 & 4 & 10 & 5 & 0 \\
\hline
\end{tabular}


A diferença de artigos, se comparada com o mapeamento feito via Google Acadêmico e Zotero, pode ser justificada por fatores como duplicação de resultados ou o mesmo artigo disponibilizado em diferentes sítios.

\subsubsection{Aplicação dos critérios de exclusão e inclusão nos artigos identificados}

Foram aplicados os critérios de exclusão e inclusão da tabela 2 nos 104 artigos encontrados (conforme o item anterior). A análise de cada artigo quanto aos critérios foi feita com base na leitura do título, resumo e palavras-chave, e, quando necessário, da sessão de materiais e métodos (e equivalentes).

Tabela 2: Critérios de exclusão e inclusão utilizados na revisão sistemática

\begin{tabular}{|l|l|}
\hline \multicolumn{1}{|c|}{ Critérios de exclusão } & \multicolumn{1}{|c|}{ Critérios de inclusão } \\
\hline $\begin{array}{l}\text { E1. Artigos no formato de revisão sistemática. } \\
\text { E2. Artigos com análise técnica de ferramentas, sem } \\
\text { descrição de uso com público-alvo, ou com propostas } \\
\text { educacionais ainda não implementadas. }\end{array}$ & $\begin{array}{l}\text { I1. Artigos publicados entre 2012 (ano de } \\
\text { lançamento da versão online do ambiente de } \\
\text { criação e compartilhamento) e 2016. } \\
\text { E3. Em casos de artigos duplicados ou similares, a } \\
\text { versão mais antiga foi desconsiderada. }\end{array}$ \\
$\begin{array}{l}\text { educacionais. } \\
\text { I3. Artigos completos (quatro ou mais } \\
\text { páginas) }\end{array}$ \\
\hline
\end{tabular}

\subsubsection{Aplicação dos critérios de avaliação nos artigos selecionados}

Após a aplicação dos critérios de inclusão e exclusão, o número de artigos foi reduzido para 53 artigos (tabela 1), sendo 21 do WIE, 13 do CBIE, 4 do SBIE, 10 da RENOTE e 5 da RBIE. Nesse conjunto de artigos, foram aplicados os critérios de avaliação baseados nas questões de pesquisa e que são apresentados na tabela 3. A análise dos critérios foi feita a partir da leitura da seção de materiais e métodos (ou equivalente) e uso de mecanismo de busca com palavras-chave, para encontrar informações sobre os critérios de avaliação que não se encontravam nessa seção em cada artigo.

Tabela 3: Critérios de avaliação utilizados na revisão sistemática

\begin{tabular}{|l|}
\hline \multicolumn{1}{|c|}{ Critérios de avaliação } \\
\hline A1. Qual o objetivo proposto da experiência relatada? \\
A2. Qual foi o público-alvo da experiência? \\
A3. Qual o tamanho do público-alvo? \\
A4. Qual o perfil do(a) condutor(a) das atividades relatadas? \\
A5. Em que ambiente as atividades foram desenvolvidas? \\
A6. Qual a duração das atividades? \\
A7. Foi utilizado o Scratch online ou offline? \\
A8. Que outras ferramentas digitais foram utilizadas? \\
A9. Quais os métodos/estratégias de avaliação utilizados na experiência? \\
\hline
\end{tabular}

\subsubsection{Leitura de artigos em destaque}

Por fim, para se investigar melhor os resultados relatados (referente à QP3), utilizaram-se os critérios de avaliação para priorizar um conjunto de artigos para leitura completa do material: considerou-se que os artigos com maior nível de descrição (itens A2 a A8) e diversidade de estratégias de avaliação (item A9) teriam maior potencial de apresentar resultados relevantes. A partir desses critérios, foram selecionados 14 para leitura detalhada, cujo processo de seleção será melhor descrito na seção 4 . 


\subsection{Ameaças à validade da pesquisa}

As principais ameaças à validade desta RLS estão relacionadas ao viés de seleção das publicações. A etapa 3.2.1 utilizou uma estratégia não usual para identificação de periódicos de busca; no entanto, o volume de artigos encontrados no mesmo na etapa seguinte reforçou a validade da estratégia. A etapa 3.2.2 foi realizada a partir de buscas manuais e mecânicas, o que pode resultar na não identificação de trabalhos por falha humana; para mitigar esse risco, a tarefa de busca foi compartilhada com outros contribuintes. Além disso, as etapas 3.2.3 e 3.2.4 tiveram também um caráter interpretativo, podendo prejudicar a extração de dados e a análise de resultados. Para reduzir esse fator, essa análise foi feita de forma colaborativa entre os autores, onde um pode contar com a revisão do outro para itens mais subjetivos.

\section{Resultados e discussão}

A partir da execução da estratégia descrita na seção anterior, foram levantados dados importantes para a formulação de respostas às questões de pesquisa definidas para essa RSL. Nesse sentido, os resultados serão aqui apresentados e discutidos com base em cada questão de pesquisa. A tabela 4 apresenta um resumo das informações obtidas, referentes aos critérios de avaliação A2 a A9 (onde S indica existência de informação e $\mathrm{N}$ indica não existência; para $\mathrm{A} 8$ e $\mathrm{A} 9$, o número indica a quantidade de ferramentas digitais e estratégias de avaliação, respectivamente) - os dados individualizados de cada uma das 53 publicações analisadas, assim como a lista com as referências bibliográficas completas, estão disponíveis em 〈https://goo.gl/va33ww〉 por limitação de espaço nesse artigo.

Tabela 4: Dados resumidos dos artigos selecionados

\begin{tabular}{|c|c|c|c|c|c|c|c|c|c|c|c|c|c|c|c|c|c|c|c|c|c|c|c|c|c|c|}
\hline artigo & A2 & A3 & A4 & A5 & A6 & A7 & A8 & A9 & Artigo & A2 & A3 & A4 & A5 & A6 & A7 & A8 & A9 & Artigo & $\mathbf{A 2}$ & A3 & $\mathbf{A 4}$ & A5 & A6 & $\begin{array}{l}\mathbf{A} 7 \\
\end{array}$ & A8 & A9 \\
\hline 1 & $\mathrm{~s}$ & $\mathrm{~s}$ & $\mathrm{~N}$ & $\mathrm{~s}$ & $\mathrm{~s}$ & $\mathrm{~N}$ & 2 & 2 & 19 & $\mathrm{~s}$ & $\mathrm{~N}$ & $\mathrm{~N}$ & $\mathrm{~N}$ & $\mathrm{~S}$ & $\mathrm{~s}$ & 0 & 2 & 37 & $\mathrm{~S}$ & $\mathrm{~N}$ & $\mathrm{~N}$ & $\mathrm{~s}$ & $\mathrm{~s}$ & $\mathrm{~N}$ & 1 & 1 \\
\hline 2 & $\mathrm{~s}$ & $\mathrm{~s}$ & $\mathrm{~N}$ & $\mathrm{~S}$ & $\mathrm{~s}$ & $\mathrm{~N}$ & 0 & 1 & 20 & $\mathrm{~S}$ & $\mathrm{~N}$ & $\mathrm{~N}$ & $\mathrm{~N}$ & $\mathrm{~s}$ & $\mathrm{~N}$ & 0 & 2 & 38 & $\mathrm{~S}$ & $\mathrm{~s}$ & $\mathrm{~N}$ & $\mathrm{~s}$ & $\mathrm{~N}$ & $\mathrm{~N}$ & 0 & 1 \\
\hline 3 & $\mathrm{~s}$ & $\mathrm{~s}$ & $\mathrm{~s}$ & $\mathrm{~s}$ & $\mathrm{~s}$ & $\mathrm{~N}$ & 4 & 1 & 21 & $\mathrm{~s}$ & $\mathrm{~s}$ & $\mathrm{~s}$ & $\mathrm{~s}$ & $\mathrm{~N}$ & $\mathrm{~s}$ & 3 & 1 & 39 & $\mathrm{~s}$ & $\mathrm{~s}$ & $\mathrm{~S}$ & $\mathrm{~s}$ & $\mathrm{~s}$ & $\mathrm{~N}$ & 0 & 1 \\
\hline 4 & $\mathrm{~S}$ & $\mathrm{~s}$ & $\mathrm{~s}$ & $\mathrm{~s}$ & $\mathrm{~s}$ & $\mathrm{~N}$ & 1 & 2 & 22 & $\mathrm{~S}$ & $\mathrm{~s}$ & $\mathrm{~N}$ & $\mathrm{~S}$ & $\mathrm{~s}$ & $\mathrm{~s}$ & 2 & 1 & 40 & $\mathrm{~s}$ & $\mathrm{~s}$ & $\mathrm{~N}$ & $\mathrm{~s}$ & $\mathrm{~s}$ & $\mathrm{~N}$ & 0 & 1 \\
\hline 5 & $\mathrm{~S}$ & $\mathrm{~s}$ & $\mathrm{~N}$ & $\mathrm{~s}$ & $\mathrm{~s}$ & $\mathrm{~N}$ & 1 & 2 & 23 & $\mathrm{~S}$ & $\mathrm{~N}$ & $\mathrm{~N}$ & $\mathrm{~s}$ & $\mathrm{~s}$ & $\mathrm{~N}$ & 3 & 1 & 41 & $\mathrm{~s}$ & $\mathrm{~N}$ & $\mathrm{~N}$ & $\mathrm{~s}$ & $\mathrm{~N}$ & $\mathrm{~N}$ & 1 & 1 \\
\hline 6 & $\mathrm{~s}$ & $\mathrm{~N}$ & $\mathrm{~s}$ & $\mathrm{~s}$ & $\mathrm{~s}$ & $\mathrm{~N}$ & 0 & 1 & 24 & $\mathrm{~s}$ & $\mathrm{~s}$ & $\mathrm{~s}$ & $\mathrm{~s}$ & $\mathrm{~s}$ & $\mathrm{~N}$ & 1 & 2 & 42 & $\mathrm{~S}$ & $\mathrm{~N}$ & $\mathrm{~s}$ & $\mathrm{~s}$ & $\mathrm{~s}$ & $\mathrm{~N}$ & 1 & 1 \\
\hline 7 & $\mathrm{~S}$ & $\mathrm{~s}$ & $\mathrm{~s}$ & $\mathrm{~s}$ & $\mathrm{~s}$ & $\mathrm{~s}$ & 0 & 3 & 25 & $\mathrm{~S}$ & $\mathrm{~s}$ & $\mathrm{~N}$ & $\mathrm{~s}$ & $\mathrm{~s}$ & $\mathrm{~s}$ & 1 & 1 & 43 & $\mathrm{~s}$ & $\mathrm{~s}$ & $\mathrm{~s}$ & $\mathrm{~s}$ & $\mathrm{~s}$ & $\mathrm{~s}$ & 0 & 2 \\
\hline 8 & $\mathrm{~S}$ & $\mathrm{~s}$ & $\mathrm{~N}$ & $\mathrm{~N}$ & $\mathrm{~s}$ & $\mathrm{~N}$ & 1 & 2 & 26 & $\mathrm{~S}$ & $\mathrm{~s}$ & $\mathrm{~s}$ & $\mathrm{~s}$ & $\mathrm{~s}$ & $\mathrm{~s}$ & 0 & 1 & 44 & S & $\mathrm{s}$ & $\mathrm{s}$ & $\mathrm{s}$ & $\mathrm{s}$ & $\mathrm{s}$ & 0 & 1 \\
\hline 9 & $\mathrm{~s}$ & $\mathrm{~s}$ & $\mathrm{~s}$ & $\mathrm{~N}$ & $\mathrm{~s}$ & $\mathrm{~N}$ & 0 & 2 & 27 & $\mathrm{~s}$ & $\mathrm{~s}$ & $\mathrm{~N}$ & $\mathrm{~s}$ & $\mathrm{~s}$ & $\mathrm{~s}$ & 0 & 2 & 45 & $\mathrm{~s}$ & $\mathrm{~s}$ & $\mathrm{~s}$ & $\mathrm{~s}$ & $\mathrm{~s}$ & $\mathrm{~N}$ & 0 & 3 \\
\hline 10 & $\mathrm{~S}$ & $\mathrm{~s}$ & $\mathrm{~s}$ & $\mathrm{~N}$ & $\mathrm{~s}$ & $\mathrm{~N}$ & 0 & 1 & 28 & $\mathrm{~S}$ & $\mathrm{~s}$ & $\mathrm{~N}$ & $\mathrm{~s}$ & $\mathrm{~s}$ & $\mathrm{~N}$ & 1 & 1 & 46 & $\mathrm{~S}$ & $\mathrm{~s}$ & $\mathrm{~s}$ & $\mathrm{~s}$ & $\mathrm{~s}$ & $\mathrm{~N}$ & 1 & 2 \\
\hline 11 & $\mathrm{~S}$ & $\mathrm{~s}$ & $\mathrm{~N}$ & $\mathrm{~S}$ & $\mathrm{~s}$ & $\mathrm{~N}$ & 2 & 1 & 29 & $\mathrm{~S}$ & $\mathrm{~N}$ & $\mathrm{~s}$ & $\mathrm{~S}$ & $\mathrm{~N}$ & $\mathrm{~N}$ & 4 & 1 & 47 & $\mathrm{~S}$ & $\mathrm{~s}$ & $\mathrm{~s}$ & $\mathrm{~s}$ & $\mathrm{~s}$ & $\mathrm{~N}$ & 0 & 2 \\
\hline 12 & $\mathrm{~S}$ & $\mathrm{~s}$ & $\mathrm{~s}$ & $\mathrm{~s}$ & $\mathrm{~s}$ & $\mathrm{~s}$ & 0 & 1 & 30 & $\mathrm{~S}$ & $\mathrm{~s}$ & $\mathrm{~N}$ & $\mathrm{~N}$ & $\mathrm{~s}$ & $\mathrm{~s}$ & 0 & 1 & 48 & $\mathrm{~S}$ & $\mathrm{~N}$ & $\mathrm{~s}$ & $\mathrm{~s}$ & $\mathrm{~N}$ & $\mathrm{~N}$ & 0 & 0 \\
\hline 13 & $\mathrm{~S}$ & $\mathrm{~S}$ & $\mathrm{~S}$ & $\mathrm{~N}$ & $\mathrm{~N}$ & $\mathrm{~N}$ & 0 & 1 & 31 & $\mathrm{~S}$ & $\mathrm{~s}$ & $\mathrm{~s}$ & $\mathrm{~N}$ & $\mathrm{~S}$ & $\mathrm{~N}$ & 1 & 2 & 49 & $\mathrm{~S}$ & $\mathrm{~S}$ & $\mathrm{~s}$ & $\mathrm{~S}$ & $\mathrm{~S}$ & $\mathrm{~s}$ & 1 & 1 \\
\hline 14 & $\mathrm{~S}$ & $\mathrm{~s}$ & $\mathrm{~N}$ & $\mathrm{~s}$ & $\mathrm{~s}$ & $\mathrm{~N}$ & 2 & 2 & 32 & $\mathrm{~S}$ & $\mathrm{~s}$ & $\mathrm{~s}$ & $\mathrm{~s}$ & $\mathrm{~s}$ & $\mathrm{~N}$ & 1 & 0 & 50 & $\mathrm{~S}$ & $\mathrm{~s}$ & $\mathrm{~s}$ & $\mathrm{~N}$ & $\mathrm{~s}$ & $\mathrm{~N}$ & 0 & 1 \\
\hline 15 & $\mathrm{~S}$ & $\mathrm{~N}$ & $\mathrm{~s}$ & $\mathrm{~N}$ & $\mathrm{~N}$ & $\mathrm{~N}$ & 1 & 2 & 33 & $\mathrm{~S}$ & $\mathrm{~s}$ & $\mathrm{~s}$ & $\mathrm{~s}$ & $\mathrm{~s}$ & $\mathrm{~N}$ & 4 & 2 & 51 & $\mathrm{~S}$ & $\mathrm{~s}$ & $\mathrm{~s}$ & $\mathrm{~s}$ & $\mathrm{~s}$ & $\mathrm{~s}$ & 0 & 2 \\
\hline 16 & $\mathrm{~S}$ & $\mathrm{~s}$ & $\mathrm{~s}$ & $\mathrm{~s}$ & $\mathrm{~S}$ & $\mathrm{~N}$ & 0 & 2 & 34 & $\mathrm{~S}$ & $\mathbf{S}$ & $\mathrm{N}$ & $\mathrm{s}$ & $\mathrm{S}$ & $\mathrm{N}$ & 0 & 2 & 52 & $\mathrm{~S}$ & $\mathrm{~s}$ & $\mathrm{~s}$ & $\mathrm{~N}$ & $\mathrm{~s}$ & $\mathrm{~N}$ & 0 & 1 \\
\hline 17 & $\mathrm{~S}$ & $\mathrm{~s}$ & $\mathrm{~s}$ & $\mathrm{~s}$ & $\mathrm{~N}$ & $\mathrm{~s}$ & 0 & 0 & 35 & $\mathrm{~S}$ & $\mathrm{~N}$ & $\mathrm{~N}$ & $\mathrm{~s}$ & $\mathrm{~N}$ & $\mathrm{~N}$ & 0 & 1 & 53 & $\mathrm{~S}$ & $\mathrm{~s}$ & $\mathrm{~N}$ & $\mathrm{~s}$ & $\mathrm{~s}$ & $\mathrm{~N}$ & 0 & 1 \\
\hline 18 & $\mathrm{~S}$ & $\mathrm{~s}$ & $\mathrm{~s}$ & $\mathrm{~s}$ & $\mathrm{~s}$ & $\mathrm{~s}$ & 0 & 1 & 26 & $\mathrm{~S}$ & S & $\mathrm{N}$ & $\mathrm{N}$ & $\mathrm{s}$ & $\mathrm{N}$ & 0 & 1 & & & & & & & & & \\
\hline
\end{tabular}

\subsection{QP1: Quais são os objetivos de uso do Scratch nas pesquisas relatadas?}

Para responder a essa pergunta, foram mapeados os objetivos descritos nos 53 artigos selecionados, conforme critério de avaliação A1. Como os objetivos são normalmente descritos por meio de frases ou parágrafos, optou-se por identificar as palavras que aparecem com mais frequência, conforme descrito no gráfico 1 .

Os resultados obtidos indicam uma tendência notada nos objetivos dos artigos: o foco no ensino ou aprendizagem de programação, computação ou pensamento computacional, utilizando o Scratch como ferramenta para tal. Apesar de ser um resultado 
esperado e condizente com os trabalhos relacionados, nota-se que o uso do Scratch como recurso para desenvolvimento de habilidades não é tão presente como um objetivo central; a criatividade, por exemplo, que normalmente é associado ao Scratch por seus criadores, não se mostra como um objetivo de pesquisa nos trabalhos selecionados.

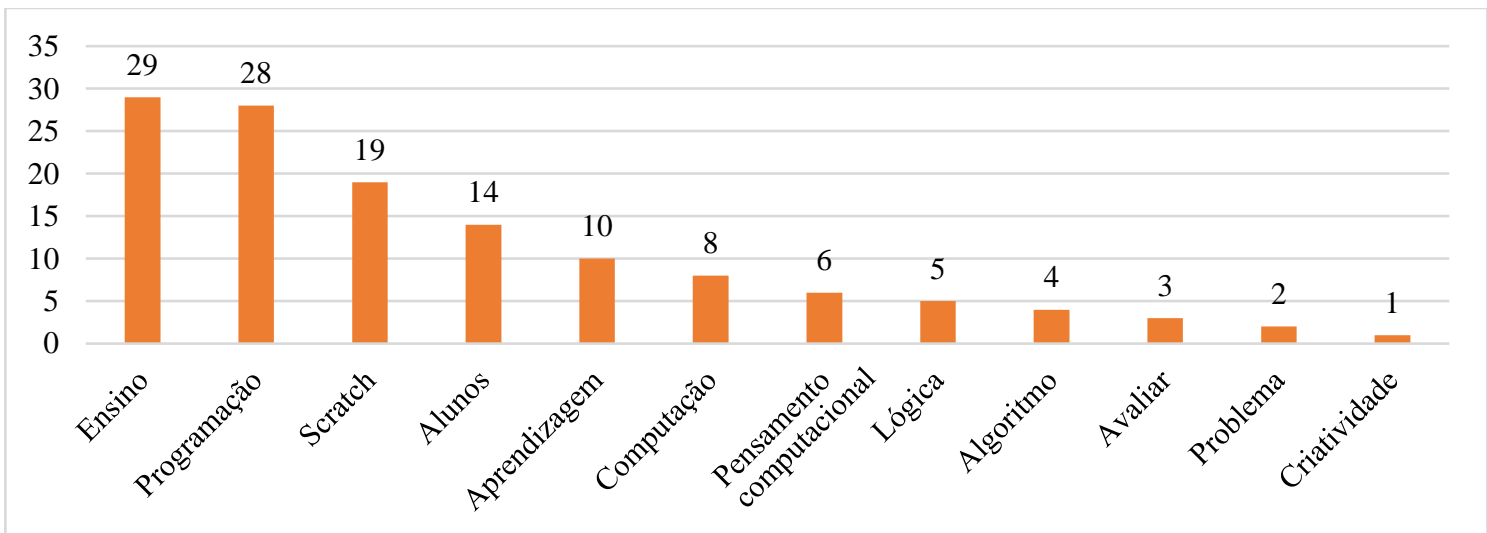

Gráfico 1: Termos utilizados na descrição dos objetivos dos artigos selecionados

\subsection{QP2: Quais são os contextos de uso e público-alvo relatados?}

Os critérios de avaliação A2 a A8 trouxeram insumos que contribuíram para a descrição dos contextos de uso do Scratch com fins educacionais. Como pode ser notado na tabela 4, em relação ao perfil do(a) aplicador(a) (A4) e ao uso online ou offline do Scratch (A7), não foi possível identificar tais informações na maioria dos arquivos analisados; dessa forma, optou-se por não realizar análise em relação a esses itens.

Em relação ao público-alvo das experiências relatadas, o gráfico 2 apresenta um resumo das informações coletadas nos 53 artigos. É importante ressaltar que em alguns artigos havia públicos de diferentes níveis escolares (por isso a amostra é maior que 53). O público dominante (ensino fundamental) condiz com o faixa etária para o qual o Scratch é destinado (8 a 16 anos), que também inclui parte do público de ensino médio. Vale ressaltar, por outro lado, o número de experiências com o ensino superior, explorando, em geral, o Scratch como ferramenta de introdução à programação, e algumas iniciativas focadas na formação de educadores para o tema.

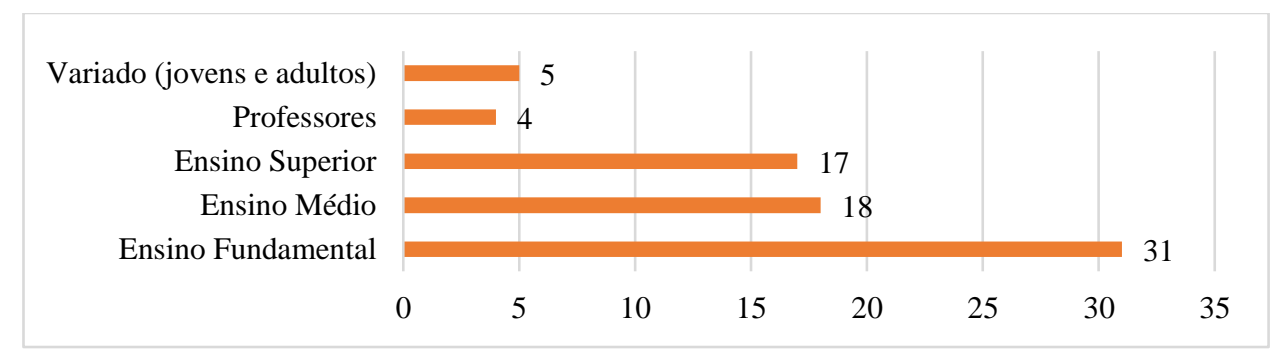

Gráfico 2: público-alvo das experiências relatadas

Quanto ao tamanho do público envolvido (A3), a maior parte das atividades ocorreu para um público entre 11 e 30 pessoas (34\%), tamanho típico de uma turma de alunos de um determinado nível escolar; poucas experiências envolveram um público que indicasse algum objetivo de escalar as atividades (6\% com mais de 100 pessoas).

A duração das atividades (A6) concentrou-se na faixa de até 20 horas de duração (60\%), o que, em um ambiente escolar, representaria 1 hora de atividade ao longo de um semestre; $26 \%$ duraram até 4 horas, podendo ser sessões realizadas de forma pontual. $O$ espaço de aplicação são, em maioria, laboratórios de informática de escolas (39\%), sendo 
$85 \%$ desse número referente a escolas públicas. Outro espaço que se destaca são os ambientes universitários (24\%), incluindo o já esperado público do ensino superior, mas também atividades de extensão e pesquisa universitária incluindo públicos externos.

Por fim, a maioria dos contextos de aplicação relatados utilizou somente o Scratch (57\%) como recurso digital para implementação da proposta dos trabalhos. Um total de outras 22 ferramentas foi identificado, dentre as quais se destacam o Arduino ( 7 relatos de uso, ou cerca de 13\%). Além de indicar que o Scratch tem se mostrado suficiente para alcançar o que é proposto em boa parte dos trabalhos com a ferramenta, pode-se considerar que o uso do Arduino nos mesmos contextos pode ser explicado como ferramenta que ajude a conectar as construções feitas no Scratch com o mundo físico.

\subsection{QP3: Quais são as estratégias de avaliação e os resultados relatados?}

A partir da leitura das sessões de materiais e métodos dos 53 artigos selecionados, foi possível identificar as principais estratégias de avaliação relatadas (A9), cuja síntese é apresentada no gráfico 3. Notou-se que em boa parte dos trabalhos foram utilizados questionários, provavelmente por sua facilidade de customização e direcionamento para a hipótese proposta. Além disso, destaca-se também a análise técnica dos artefatos construídos pelos participantes como estratégia de avaliação, o que, apesar de exigir maior conhecimento do Scratch em si, permite inferir o uso de conceitos e práticas relacionadas à programação e ao pensamento computacional.

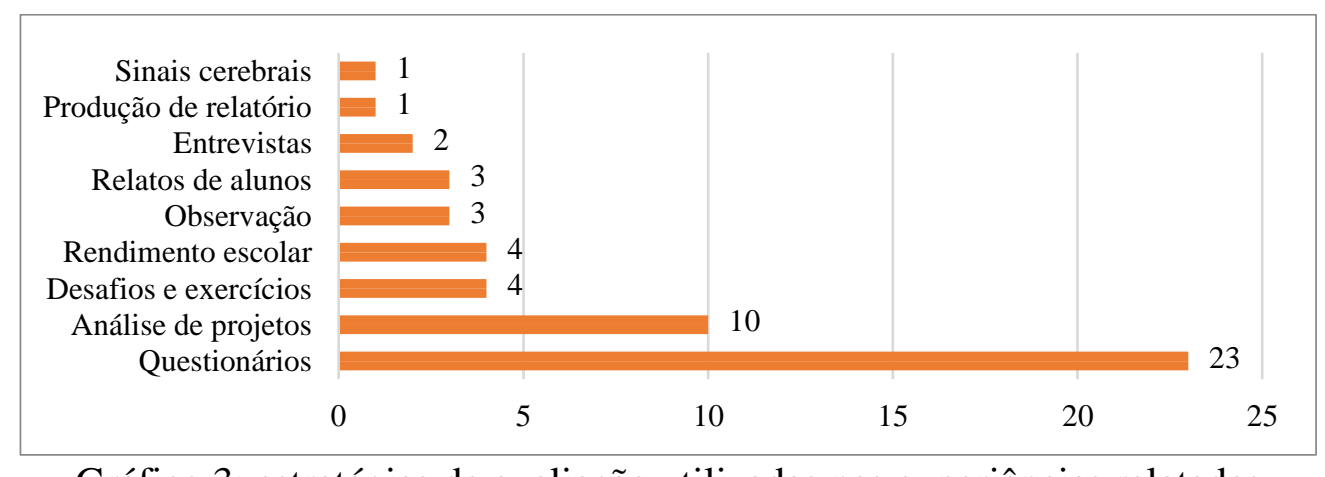

Gráfico 3: estratégias de avaliação utilizadas nas experiências relatadas

Por fim, o número de estratégias de avaliação (A9) indicadas em cada artigo, que variou entre 0 e 3, e o nível de descrição da experiência - informações referentes a público (A2 e A3), espaço de aplicação (A5) e duração (A6) - foram utilizados para ponderar quais artigos seriam priorizados para leitura detalhada dos resultados. Nesse formato, os artigos foram pontuados de 0 a 7 e 14 deles (pontuação 6 e 7) foram selecionados; a partir de sua leitura, a tabela 5 apresenta uma breve descrição desses trabalhos e os principais resultados obtidos, evidenciando a variedade de conclusões obtidas - algo já sinalizado pela falta de padrão de estratégias de avaliação.

Tabela 5: Principais resultados dos artigos selecionados

\begin{tabular}{|l|l|l|}
\hline \multicolumn{1}{|c|}{ Trabalho } & \multicolumn{1}{|c|}{ Descrição ou objetivo } & \multicolumn{1}{c|}{ Principais resultados } \\
\hline $\begin{array}{l}\text { Da Cruz } \\
\text { Alves et al. } \\
(2016)\end{array}$ & $\begin{array}{l}\text { Unidade instrucional para o ensino de } \\
\text { computação no Ensino Fundamental de } \\
\text { forma multidisciplinar. }\end{array}$ & $\begin{array}{l}\text { Mudança significativa na sua percepção } \\
\text { sobre a capacidade de programar, além } \\
\text { de alto nível de motivação durante as } \\
\text { atividades. }\end{array}$ \\
\hline $\begin{array}{l}\text { Von } \\
\text { Wangenheim, } \\
\text { Nunes e Dos } \\
\text { Santos (2014) }\end{array}$ & $\begin{array}{l}\text { Unidade instrucional para o ensino de } \\
\text { computação no Ensino Fundamental de } \\
\text { forma interdisciplinar. }\end{array}$ & $\begin{array}{l}\text { Vontade de continuar programando, } \\
\text { além de reforço de competências de } \\
\text { interpretação de histórias e compreensão } \\
\text { da sequência das ações. }\end{array}$ \\
\hline
\end{tabular}




\begin{tabular}{|c|c|c|}
\hline $\begin{array}{l}\text { Lopes et al. } \\
\text { (2016) }\end{array}$ & $\begin{array}{l}\text { Ensino de Algoritmos e Lógica de } \\
\text { Programação, com foco na criação de } \\
\text { programas para aplicação de conceitos } \\
\text { matemáticos. }\end{array}$ & $\begin{array}{l}\text { Impacto positivo no rendimento em } \\
\text { Matemática, aumento do interesse de } \\
\text { alunos nas áreas de Matemática e } \\
\text { Computação. }\end{array}$ \\
\hline $\begin{array}{l}\text { Da Rocha } \\
(2015)\end{array}$ & $\begin{array}{l}\text { Estudo de caso sobre como os alunos } \\
\text { constroem o conceito de ângulo via } \\
\text { programação. }\end{array}$ & $\begin{array}{l}\text { Melhorias em termos de engajamento } \\
\text { dos alunos e significação do conceito de } \\
\text { ângulos. }\end{array}$ \\
\hline $\begin{array}{l}\text { Barcelos, } \\
\text { Bortoletto e } \\
\text { Andrioli } \\
(2016)\end{array}$ & $\begin{array}{l}\text { Formação de professores de Matemática } \\
\text { para criação de atividades que envolvam } \\
\text { tópicos matemáticos e desenvolvimento } \\
\text { de competências do Pensamento } \\
\text { Computacional. }\end{array}$ & $\begin{array}{l}\text { Maior engajamento em atividades } \\
\text { práticas de construção de artefatos. }\end{array}$ \\
\hline $\begin{array}{l}\text { Da Silva, De } \\
\text { Araújo e Da } \\
\text { Silva Aranha } \\
(2014)\end{array}$ & $\begin{array}{l}\text { Oportunidade, para educadores, de } \\
\text { utilização do software Scratch como } \\
\text { ferramenta auxiliar na prática docente. }\end{array}$ & $\begin{array}{l}\text { Grande número de interessados em } \\
\text { utilizar o Scratch em sala de aula após } \\
\text { algumas oficinas de programação (87\%), } \\
\text { especialmente da área de Matemática. }\end{array}$ \\
\hline $\begin{array}{l}\text { Dos Santos } \\
\text { Silva, De } \\
\text { Souza } \\
\text { Moraes e } \\
\text { Batista } \\
(2014) \\
\end{array}$ & $\begin{array}{l}\text { Análise da contribuição de objetos de } \\
\text { aprendizagem desenvolvidos no Scratch } \\
\text { ao serem utilizados como ferramentas } \\
\text { pedagógicas para o ensino de Saneamento } \\
\text { Básico. }\end{array}$ & $\begin{array}{l}\text { Apropriação e aumento do repertório dos } \\
\text { alunos perante a temática de saneamento }\end{array}$ \\
\hline $\begin{array}{l}\text { Garcia et al. } \\
(2016)\end{array}$ & $\begin{array}{l}\text { Ensino de programação de forma simples } \\
\text { e motivadora, com a finalidade de } \\
\text { despertar o interesse de jovens e adultos } \\
\text { pela programação. }\end{array}$ & $\begin{array}{l}\text { Evolução técnica do público participante } \\
\text { (por meio de avaliações sobre o tema) e } \\
\text { também maior interesse por cursos de } \\
\text { ensino superior em Computação. }\end{array}$ \\
\hline $\begin{array}{l}\text { Arantes e Da } \\
\text { Silva Ferreira } \\
(2015)\end{array}$ & $\begin{array}{l}\text { Ensino dos conceitos de variáveis, } \\
\text { condicionais e concorrência de uma } \\
\text { maneira mais leve e divertida. }\end{array}$ & $\begin{array}{l}\text { Promoção da socialização e descontração } \\
\text { ao grupo, fomentando o interesse dos } \\
\text { jovens pela programação. }\end{array}$ \\
\hline $\begin{array}{l}\text { Bathke e } \\
\text { Raabe (2016) }\end{array}$ & $\begin{array}{l}\text { Estímulo ao desenvolvimento do } \\
\text { pensamento computacional em estudantes } \\
\text { da educação de jovens e adultos - EJA. }\end{array}$ & $\begin{array}{l}\text { Público com diferentes tipos de relação } \\
\text { com a tecnologia, o que demanda } \\
\text { estratégias diferenciadas. }\end{array}$ \\
\hline $\begin{array}{l}\text { De Quadro } \\
\text { Martins et al. } \\
(2016)\end{array}$ & $\begin{array}{l}\text { Estímulo à criatividade de jovens por } \\
\text { meio da robótica educacional. }\end{array}$ & $\begin{array}{l}\text { Despertar da curiosidade e da motivação } \\
\text { intrínseca dos jovens durante a } \\
\text { realização das atividades; o uso de } \\
\text { pensamento organizado e procedimental. }\end{array}$ \\
\hline $\begin{array}{l}\text { Andrade et } \\
\text { al. (2016) }\end{array}$ & $\begin{array}{l}\text { Abordagem dos aspectos } \\
\text { multidisciplinares intrínsecos ao processo } \\
\text { de desenvolvimento de jogos para } \\
\text { estimular o ensino de programação. }\end{array}$ & $\begin{array}{l}\text { Interesse de } 93 \% \text { do público pelo } \\
\text { desenvolvimento de jogos. }\end{array}$ \\
\hline $\begin{array}{l}\text { De Oliveira } \\
\text { Fernandez et } \\
\text { al. }(2015)\end{array}$ & $\begin{array}{l}\text { Oficina de Internet das Coisas estruturada } \\
\text { a partir de estratégias de Aprendizagem } \\
\text { Baseada em Projetos. }\end{array}$ & $\begin{array}{l}\text { Forte aceitação do conteúdo e formato } \\
\text { das atividades, expressos principalmente } \\
\text { nos comentários dos participantes. }\end{array}$ \\
\hline $\begin{array}{l}\text { Hayashi, } \\
\text { Posada e } \\
\text { Baranauskas } \\
(2013)\end{array}$ & $\begin{array}{l}\text { Investigação de aspectos sociais e afetivos } \\
\text { em ambientes e sistemas computacionais } \\
\text { de apoio à aprendizagem. }\end{array}$ & $\begin{array}{l}\text { Capacidade de integração do Scratch } \\
\text { com outras mídias }\end{array}$ \\
\hline
\end{tabular}

\subsection{QP4: Há instituições que se destacam nesse cenário?}

No âmbito dos 53 artigos selecionados, foram identificadas 49 instituições diferentes (um mesmo trabalho poderia ter mais de uma instituição vinculada). Havia o interesse em mapear o perfil dos grupos de pesquisa envolvidos, porém essa análise foi descartada pela quantidade significativa de artigos que não traziam essa informação. Entre as principais instituições, destacam-se UNICAMP, UFPB e IFSP, com 6, 4 e 3 trabalhos selecionados, respectivamente. Vale ressaltar, também, a representatividade regional das publicações, contendo representantes de todas as regiões do país. 


\section{Considerações finais}

Esse trabalho teve como objetivo realizar uma revisão sistemática da literatura (RSL) acerca dos trabalhos referentes ao uso da linguagem de programação Scratch com objetivos educacionais, entre os anos de 2012 e 2016. De um volume inicial de 104 artigos selecionados, 53 foram avaliados e os resultados de 14 desses artigos foram discutidos.

Como principal objetivo relatado, identificou-se uma tendência no uso do Scratch como recurso para promover o ensino-aprendizagem de lógica de programação de computadores, podendo ou não estar integrada com outras áreas do conhecimento. Quanto ao contexto de uso, as experiências analisadas são, em sua maioria, focadas em alunos do ensino fundamental, nos laboratórios de informáticas de escolas da educação básica ou de espaços universitários; além disso, identificou-se um número significativo de experiências voltadas ao ensino superior. Além disso, apesar de a maioria das experiências utilizar somente o Scratch como ferramenta digital, identificaram-se outras ferramentas associadas a este, dentre elas o Arduino.

As estratégias de avaliação utilizadas nas experiências têm, em sua maioria, explorado questionários - alguns no formato pré e pós - para extrair dados que possam ser analisados; além disso, a análise dos artefatos construídos é prática comum e buscam se amparar em propostas de outras pesquisas como parâmetro. Os resultados obtidos, apesar de diversos, são promissores no que diz respeito ao engajamento dos públicos-alvo e alguns deles já trazem indicativos de possíveis impactos que podem ser utilizados como ponto de partida para outras investigações.

Como trabalhos futuros, espera-se utilizar os aprendizados dessa RSL como ponto de partida para investigação de como apoiar um ou mais dos perfis identificados, com um interesse especial em investigar mais a fundo ações envolvendo professores da educação básica e seus desafios, especialmente nas estratégias de avaliação utilizadas.

\section{Referências}

ANDRADE, Raul et al. Uma Proposta de Oficina de Desenvolvimento de Jogos Digitais para Ensino de Programação. Anais dos Workshops do Congresso Brasileiro de Informática na Educação. 2016. p. 1127.

ARANTES, Flávia; DA SILVA FERREIRA, José Michael Leandro. Uma dinâmica para ensino de conceitos fundamentais de programação. Anais dos Workshops do Congresso Brasileiro de Informática na Educação. 2015. p. 1218.

BARCELOS, Thiago; BORTOLETTO, Rodrigo; ANDRIOLI, Mary. Formação online para o desenvolvimento do Pensamento Computacional em professores de Matemática. In: Anais dos Workshops do Congresso Brasileiro de Informática na Educação. 2016. p. 1228.

BATHKE, Julia; RAABE, André. Pensamento Computacional na Educação de Jovens e Adultos: Lições Aprendidas. Anais dos Workshops do Congresso Brasileiro de Informática na Educação. 2016. p. 1087.

BOMBASAR, James et al. Ferramentas para o ensino-aprendizagem do pensamento computacional: onde está alan turing?. Brazilian Symposium on Computers in Education (Simpósio Brasileiro de Informática na Educação-SBIE). 2015. p. 81.

DA CRUZ ALVES, Nathalia et al. Ensino de Computação de Forma Multidisciplinar em Disciplinas de História no Ensino Fundamental--Um Estudo de Caso. Revista Brasileira de Informática na Educação, v. 24, n. 3, 2016.

DA ROCHA, Kátia Coelho. PROGRAMANDO COM O SCRATCH NA AULA DE MATEMÁTICA. RENOTE, v. 13, n. 2, 2015.

DA SILVA, Thiago Reis; DE ARAUJO, Glaúber Galvão; DA SILVA ARANHA, Eduardo Henrique. Oficinas itinerantes de scratch e computação desplugada para 
professores como apoio ao ensino de computação - um relato de experiência. Anais do Workshop de Informática na Escola. 2014. p. 380.

DASGUPTA, Sayamindu; RESNICK, Mitchel. Engaging novices in programming, experimenting, and learning with data. ACM Inroads, v. 5, n. 4, p. 72-75, 2014.

DE OLIVEIRA FERNANDEZ, Cássia et al. Uma proposta baseada em projetos para oficinas de Internet das Coisas com Arduino voltadas a estudantes do Ensino Médio. RENOTE, v. 13, n. 2, 2015.

DE QUADROS MARTINS, Amilton R. et al. Use of educational robotics as a stimulus to creativity. RENOTE, v. 14, n. 1, 2016.

DOS SANTOS SILVA, Aline Marcelino; DE SOUZA MORAES, Deize Amara Silva; BATISTA, Silvia Cristina Freitas. EDUCAÇÃO AMBIENTAL: SCRATCH COMO FERRAMENTA PEDAGÓGICA NO ENSINO DE SANEAMENTO BÁSICO. RENOTE, v. 12, n. 1, 2014.

FERRI, Juliana; DOS SANTOS ROSA, Selma. Como o Ensino de Programação de Computadores Pode Contribuir Com a Construção de Conhecimento na Educação Básica Uma Revisão Sistemática da Literatura. RENOTE, v. 14, n. 2, 2016.

FOERSTER, Klaus-Tycho. Integrating Programming into the Mathematics Curriculum: Combining Scratch and Geometry in Grades 6 and 7. Proceedings of the 17th Annual Conference on Information Technology Education. ACM, 2016. p. 91-96.

GARCIA, Victor Ronchi et al. Despertando Jovens Talentos com o Conhecimento da Computação. Anais do Workshop de Informática na Escola. 2016. p. 583.

HAYASHI, Elaine CS; POSADA, Julián E. Gutiérrez; BARANAUSKAS, M. Cecília C. Explorando princípios de Afetibilidade no redesign de aplicações para contextos educacionais. Brazilian Symposium on Computers in Education (Simpósio Brasileiro de Informática na Educação-SBIE). 2013. p. 788.

KIETCHENHAM, B. A. Guidelines for performing Systematic Literature Reviews in Software Engineering. 2007.

LOPES, Claudivan Cruz et al. O Ensino de Algoritmos e Lógica de Programação como uma Ferramenta Pedagógica para Auxiliar a Aprendizagem de Matemática: Um Relato de Experiência. Anais do Workshop de Informática na Escola. 2016. p. 41.

MALAN, David J.; LEITNER, Henry H. Scratch for budding computer scientists. ACM SIGCSE Bulletin, v. 39, n. 1, p. 223-227, 2007.

MORENO-LEÓN, Jesús; ROBLES, Gregorio. Code to learn with Scratch? A systematic literature review. Global Engineering Education Conference (EDUCON), 2016 IEEE. IEEE, 2016. p. 150-156.

PAPERT, Seymour. Mindstorms: Children, computers, and powerful ideas. Basic Books, Inc., 1980.

RESNICK, Mitchel et al. Scratch: programming for all. Communications of the ACM, v. 52, n. 11, p. 60-67, 2009.

SCRATCH. Disponível em: 〈https://scratch.mit.edu/>. Acesso em 05 de mai. de 2017. SOUZA, Saymon; CASTRO, Thais. Investigação em programação com Scratch para crianças: uma revisão sistemática da literatura. Anais dos Workshops do Congresso Brasileiro de Informática na Educação. 2016. p. 1078.

VIHAVAINEN, Arto; AIRAKSINEN, Jonne; WATSON, Christopher. A systematic review of approaches for teaching introductory programming and their influence on success. Proceedings of the tenth annual conference on International computing education research. ACM, 2014. p. 19-26.

VON WANGENHEIM, Christiane Gresse; NUNES, Vinícius Rodrigues; DOS SANTOS, Giovane Daniel. Ensino de computação com scratch no ensino fundamental-um estudo de caso. Revista Brasileira de Informática na Educação, v. 22, n. 03, p. 115, 2014. 